\title{
Development of a clinical decision support system for severity risk prediction and triage of COVID-19 patients at hospital admission: an international multicentre study
}

\author{
Guangyao $\mathrm{Wu}^{1,17,18}$, Pei Yang ${ }^{2,17}$, Yuanliang Xie ${ }^{2}$, Henry C. Woodruff (1) 1,3, \\ Xiangang Rao ${ }^{4}$, Julien Guiot $\mathbb{1}^{5}$, Anne-Noelle Frix ${ }^{5}$, Renaud Louis ${ }^{5}$, \\ Michel Moutschen ${ }^{6}$, Jiawei $\mathrm{Li}^{7}$, Jing $\mathrm{Li}^{8}$, Chenggong Yan ${ }^{1,9}$, Dan $\mathrm{Du}^{2}$, \\ Shengchao Zhao ${ }^{2}$, Yi Ding $^{2}$, Bin Liu ${ }^{2}$, Wenwu Sun ${ }^{10}$, Fabrizio Albarello ${ }^{11}$, \\ Alessandra D'Abramo ${ }^{11}$, Vincenzo Schininà ${ }^{11}$, Emanuele Nicastri ${ }^{11}$, \\ Mariaelena Occhipinti ${ }^{12}$, Giovanni Barisione (1) ${ }^{13}$, Emanuela Barisione (1) ${ }^{14}$, \\ Iva Halilaj ${ }^{1}$, Pierre Lovinfosse ${ }^{15}$, Xiang Wang ${ }^{2,18}$, Jianlin $\mathrm{Wu}^{16}$ and \\ Philippe Lambin (10) ${ }^{1,3}$
}

@ERSpublications

An internationally validated model, nomogram, and online calculator for severity risk assessment and triage of COVID-19 patients at hospital admission https://bit.ly/2BQfXFs

Cite this article as: Wu G, Yang P, Xie Y, et al. Development of a clinical decision support system for severity risk prediction and triage of COVID-19 patients at hospital admission: an international multicentre study. Eur Respir J 2020; 56: 2001104 [https://doi.org/10.1183/13993003.01104-2020].

\section{ABSTRACT}

Background: The outbreak of coronavirus disease 2019 (COVID-19) has globally strained medical resources and caused significant mortality.

Objective: To develop and validate a machine-learning model based on clinical features for severity risk assessment and triage for COVID-19 patients at hospital admission.

Method: 725 patients were used to train and validate the model. This included a retrospective cohort from Wuhan, China of 299 hospitalised COVID-19 patients from 23 December 2019 to 13 February 2020, and five cohorts with 426 patients from eight centres in China, Italy and Belgium from 20 February 2020 to 21 March 2020. The main outcome was the onset of severe or critical illness during hospitalisation. Model performances were quantified using the area under the receiver operating characteristic curve (AUC) and metrics derived from the confusion matrix.

Results: In the retrospective cohort, the median age was 50 years and 137 (45.8\%) were male. In the five test cohorts, the median age was 62 years and 236 (55.4\%) were male. The model was prospectively validated on five cohorts yielding AUCs ranging from 0.84 to 0.93 , with accuracies ranging from $74.4 \%$ to $87.5 \%$, sensitivities ranging from $75.0 \%$ to $96.9 \%$, and specificities ranging from $55.0 \%$ to $88.0 \%$, most of which performed better than the pneumonia severity index. The cut-off values of the low-, medium- and high-risk probabilities were 0.21 and 0.80 . The online calculators can be found at www.covid19risk.ai.

Conclusion: The machine-learning model, nomogram and online calculator might be useful to access the onset of severe and critical illness among COVID-19 patients and triage at hospital admission.

This article has supplementary material available from erj.ersjournals.com

Received: 9 April 2020 | Accepted after revision: 11 June 2020

Copyright OERS 2020. This version is distributed under the terms of the Creative Commons Attribution NonCommercial Licence 4.0. 


\section{Introduction}

In December 2019, a novel coronavirus, severe acute respiratory syndrome-coronavirus-2 (SARS-CoV-2; earlier named as 2019-nCoV), emerged in Wuhan, China [1]. The disease caused by SARS-CoV-2 was named coronavirus disease 2019 (COVID-19). As of 15 May 2020, more than 4490000 COVID-19 patients have been reported globally, and over 300000 patients have died [2]. The outbreak of COVID-19 has developed into a pandemic [3].

Among COVID-19 patients, around 80\% present with mild illness whose symptoms usually disappear within 2 weeks [4]. However, around 20\% of the patients may proceed and necessitate hospitalisation and increased medical support. The mortality rate for severe patients is around $13.4 \%$ [4]. Therefore, risk assessment of patients preferably in a quantitative, non-subjective way is extremely important for patient management and medical resource allocation. General quarantine and symptomatic treatment at home or mobile hospitals can be used for most non-severe patients, while a higher level of care and fast track to the intensive care unit (ICU) is needed for severe patients. Previous studies have summarised the clinical and radiological characteristics of severe COVID-19 patients, while the prognostic value of different variables is still unclear $[5,6]$.

Several scoring systems that are in common clinical use (e.g. Sequential Organ Failure Assessment score; Confusion, Urea, Respiratory rate, Blood pressure, Age 65; Acute Physiology and Chronic Health Evaluation, etc.) could be applied to the triage problem, albeit each with their own problems and limitations, such as the need for laboratory variables that are hard to obtain at hospital admission [7]. The pneumonia severity index (PSI) stands out as it is used to assess the probability of severity and mortality among adult patients with community-acquired pneumonia and to help hospitalisation management [8].

A better solution could possibly be found using machine learning, a branch of artificial intelligence that learns from past data in order to build a prognostic model [9]. In recent years, machine learning has been developed as a useful tool to analyse large amounts of data from medical records or images [10]. Previous modelling studies focused on forecasting the potential international spread of COVID-19 [11].

Therefore, our objective is to develop and validate a prognostic machine-learning model based on clinical, laboratory and radiological variables of COVID-19 patients at hospital admission for severity risk assessment during hospitalisation, and compare the performance with that of PSI as a representative clinical assessment method. Our ambition is to develop a multifactorial decision support system with different datasets to facilitate risk prediction and triage (home or mobile hospital quarantine, hospitalisation or ICU) of the patient at hospital admission.

\section{Methods}

\section{Patients}

The institutional review board approved this study (2020-71), which followed the Standards for Reporting of Diagnostic Accuracy Studies statement [12], and the requirement for written informed consent was waived. 299 adult confirmed COVID-19 patients from the central hospital of Wuhan, China were included consecutively and retrospectively between 23 December 2019 and 13 February 2020. The inclusion criteria were: 1) patients with confirmed COVID-19 disease; and 2) patients presenting at hospital for admission. The exclusion criteria were: 1) patients who already had a severe illness at hospital admission; 2) time interval $>2$ days between admission and examinations; and 3) no data available or delayed results as described

Affiliations: ${ }^{1}$ The D-Lab, Dept of Precision Medicine, GROW - School for Oncology, Maastricht University Medical Center+, Maastricht, The Netherlands. ${ }^{2}$ Dept of Radiology, The Central Hospital of Wuhan, Huazhong University of Science and Technology, Wuhan, China. ${ }^{3}$ Dept of Radiology and Nuclear Medicine, GROW- School for Oncology and Developmental Biology, Maastricht University Medical Center+. Maastricht, The Netherlands. ${ }^{4}$ Dept of Ultrasound, The Central Hospital of Huangshi, Huangshi, China. ${ }^{5}$ Dept of Respiratory Medicine, CHU of Liège, Liège, Belgium. ${ }^{6}$ Dept of Infectiology, CHU of Liège, Liège, Belgium. ${ }^{7}$ Dept of Radiology, China Resources Wuhan Iron and Steel Hospital, Wuhan, China. ${ }^{8}$ Dept of Radiology, The Central Hospital of Shaoyang, Shaoyang, China. ${ }^{9}$ Dept of Medical Imaging Center, Nanfang Hospital, Southern Medical University, Guangzhou, China. ${ }^{10}$ Dept of Intensive Care Unit, The Central Hospital of Wuhan, Huazhong University of Science and Technology, Wuhan, China. ${ }^{11}$ National Institute for Infectious Diseases - IRCCS, Rome, Italy. ${ }^{12}$ Dept of Biomedical, Clinical and Experimental Sciences "Mario Serio", University of Florence, Florence, Italy. ${ }^{13}$ Unit of Respiratory Pathophysiology, Respiratory Diseases and Allergy Clinic, Dept of Internal Medicine and Medical Specialties, University of Genoa, IRCCS Ospedale Policlinico San Martino, Genoa, Italy. ${ }^{14}$ Unit of Interventional Pulmonology, IRCCS Ospedale Policlinico San Martino, Genoa, Italy. ${ }^{15}$ Nuclear Medicine and Oncological Imaging. Department of Medical Physics, CHU of Liège, Liège, Belgium. ${ }^{16}$ Dept of Radiology, Affiliated Zhongshan Hospital of Dalian University, Dalian, China. ${ }^{17}$ Guangyao Wu and Pei Yang are joint first authors. ${ }^{18}$ Guangyao Wu and Xiang Wang are co-corresponding authors.

Correspondence: Guangyao Wu, The D-Lab, Dept of Precision Medicine, GROW - School for Oncology, Maastricht University Medical Center+, 6229 ER, Maastricht, The Netherlands. E-mail: g.wudamaastrichtuniversity.nl 
below. The patients included from this centre were divided into two datasets according to the entrance time of hospitalisation, $80 \%$ for training (239 patients from 23 December 2019 to 28 January 2020) and 20\% for internal validation (60 patients from 29 January to 13 February 2020). The five test datasets were collected between 20 February 2020 and 31 March 2020 from other eight centres (supplementary material) in China, Italy and Belgium under the same inclusion and exclusion criteria (figure 1).

Patients were labelled as having a "severe disease" if at least one of the following criteria were met during hospitalisation [6,13]: 1) respiratory failure requiring mechanical ventilation; 2) shock; 3) ICU admission; 4) organ failure; or 5) death. Patients were labelled as having a "non-severe disease" if none of the above mentioned criteria were met during the whole hospitalisation process until deemed recovered and discharged from the hospital.

\section{Data collection}

Clinical, laboratory and radiological characteristics, and outcome data were obtained from the case record form shared by the International Severe Acute Respiratory and Emerging Infection Consortium from the electronic medical records [14]. A confirmed case with COVID-19 was defined as a positive result of high-throughput sequencing or real-time reverse-transcriptase PCR assay for nasal and pharyngeal swab specimens. After consultation with respiratory specialists and review of the recent COVID-19 literature, a set of clinical, laboratory and radiological characteristics was identified and data collected from the electronic medical system. The clinical characteristics included basic information (five variables), comorbidities (11 variables) and symptoms (13 variables). All clinical characteristics were obtained when the patients were admitted to hospital for the first time. 42 laboratory results were recorded, including complete blood count, white blood cell differential count, D-dimer, C-reactive protein (CRP), cardiac enzymes, procalcitonin, liver function test, kidney function test, B-type natriuretic peptide and electrolyte test. The arterial blood gas was not taken into account due to missing data for most early-stage patients. The metric conversion of laboratory results was performed using an online conversion table [15]. A detailed list of variables can be found in tables 1 and 2 .

The semantic computed tomography (CT) characteristics (including ground-glass opacity, consolidation, vascular enlargement, air bronchogram and lesion range score) were independently evaluated on all datasets by two radiologists (P. Yang, a radiologist with 5 years' experience in chest CT images, and Y. Xie, a radiologist with 20 years' experience in chest CT images), who were blinded to clinical and laboratory results. Any disagreement was resolved by a consensus read. Lesion range was identified as areas of ground-glass opacity or consolidation and was graded with a six-point scale according to the lesion volume proportion in each single lobe: $0=$ no lung parenchyma involved; $1=$ up to $5 \%$ of lung parenchyma

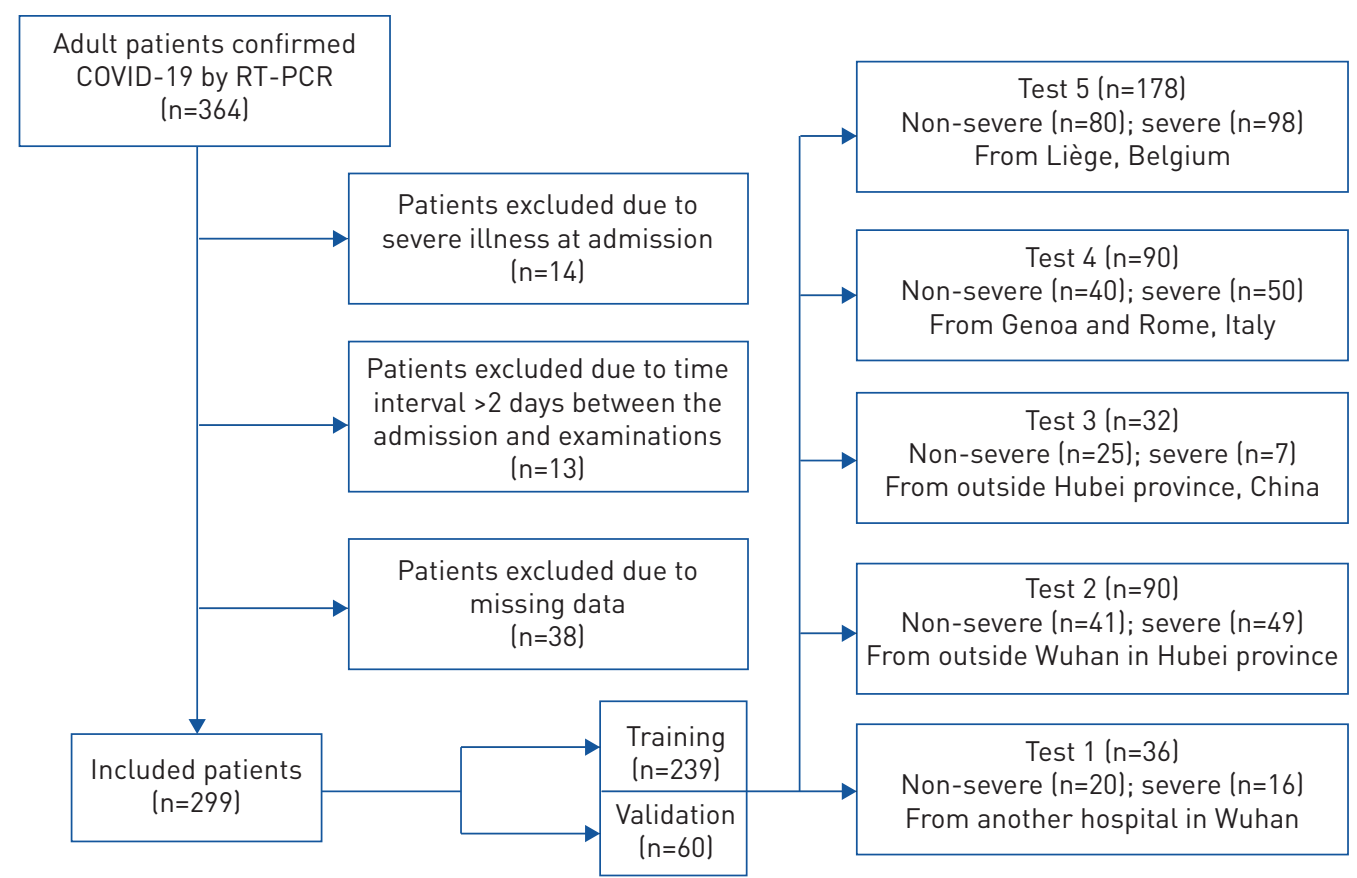

FIGURE 1 Flowchart of the patient selection process. COVID-19: coronavirus disease 2019; RT-PCR: reverse transcriptase-PCR. 


\begin{tabular}{|c|c|c|c|}
\hline & Non-severe group & Severe group & p-value ${ }^{\#}$ \\
\hline Subjects $\mathrm{n}$ & 228 & 71 & \\
\hline Age years & $43.0(33.0-61.0)$ & $62.0(52.5-71.5)$ & $<0.001$ \\
\hline Males & $95(41.7)$ & $42(59.2)$ & 0.014 \\
\hline Smoking history & $17(7.5)$ & $14(19.7)$ & 0.006 \\
\hline Hospital staff & 86 (37.7) & $4(5.6)$ & $<0.001$ \\
\hline Time of onset of illness days & $4.0(2.0-7.0)$ & $4.0(2.0-7.0)$ & 0.963 \\
\hline \multicolumn{4}{|l|}{ Comorbidities } \\
\hline Hypertension & 44 (19.3) & $31(43.7)$ & $<0.001$ \\
\hline Diabetes & 19 (8.3) & $18(25.4)$ & $<0.001$ \\
\hline Hyperlipidaemia & $11(4.8)$ & $5(7.0)$ & 0.545 \\
\hline Cardiopathy disease & $2(0.88)$ & 8 (11.3) & $<0.001$ \\
\hline COPD & 8 (3.5) & $13(18.3)$ & $<0.001$ \\
\hline Cerebrovascular disease & $6(2.6)$ & $16(22.5)$ & $<0.001$ \\
\hline Kidney disease & $5(2.2)$ & $11(15.5)$ & $<0.001$ \\
\hline Fatty liver & $28(12.3)$ & $12(16.9)$ & 0.322 \\
\hline Hepatitis B virus carrier & $2(0.88)$ & $5(7.0)$ & 0.009 \\
\hline Cancer history & $12(5.3)$ & $4(5.6)$ & 1 \\
\hline Surgical history & $30(13.2)$ & $15(21.1)$ & 0.127 \\
\hline \multicolumn{4}{|l|}{ Symptoms } \\
\hline Fever & $172(75.4)$ & 47 (66.2) & 0.128 \\
\hline Body temperature $\mathrm{C}$ & $37.8(37.3-38.4)$ & $37.5(36.8-38.0)$ & 0.027 \\
\hline Cough & $153(67.1)$ & $46(64.8)$ & 0.774 \\
\hline Sputum & $59(25.9)$ & $25(35.2)$ & 0.133 \\
\hline Weakness & $102(44.7)$ & $31(43.7)$ & 0.892 \\
\hline Diarrhoea & $25(11.0)$ & 8 (11.3) & 1 \\
\hline Vomiting & $16(7.0)$ & $9(12.7)$ & 0.144 \\
\hline Chest tightness & 50 (21.9) & $30(42.3)$ & 0.001 \\
\hline Dyspnoea & $11(4.8)$ & $4(5.6)$ & 0.760 \\
\hline Muscular soreness & $61(26.8)$ & $17(23.9)$ & 0.757 \\
\hline Chill & $38(16.7)$ & $11(15.5)$ & 1 \\
\hline Conjunctival congestion & $1(0.44)$ & $1(1.4)$ & 0.419 \\
\hline Headache or dizziness & $34(14.9)$ & $12(16.9)$ & 0.708 \\
\hline \multicolumn{4}{|l|}{ Radiological findings } \\
\hline Main findings & & & 0.928 \\
\hline Normal & $4(1.8)$ & $1(1.4)$ & \\
\hline Ground-glass opacity only & $132(57.9)$ & 39 (54.9) & \\
\hline Consolidation only & $22(9.6)$ & $6(8.5)$ & \\
\hline Mixed & 70 (30.7) & $25(35.2)$ & \\
\hline Vascular enlargement & 66 (28.9) & $36(50.7)$ & $<0.001$ \\
\hline Air bronchogram & 49 (21.5) & 30 (42.3) & 0.001 \\
\hline Lesion range score & $4.5(2.0-7.0)$ & $6.0(4.0-10.5)$ & 0.001 \\
\hline
\end{tabular}

Data are presented median (interquartile range) or $\mathrm{n}(\%)$, unless otherwise stated. \#: obtained using Fisher's exact test or Mann-Whitney U-test.

involved; $2=5-25 \% ; 3=26-50 \% ; 4=51-75 \%$; and $5=76-100 \%$ of lung parenchyma involved. The lesion volume proportion was automatically calculated by the Pneumonia Assisted Diagnosis System (version 1.17.0; Shukun Technology), and the final score was the total score from five lobes (figure 2). Detailed CT acquisition and reconstruction parameters are presented in the supplementary material.

Feature selection and modelling

All feature selection and model training were performed in the training dataset alone to prevent information leakage. An overview of the functions used is given in table S1. In order to reduce feature dimensionality, features showing high pairwise Spearman correlation $(r=>0.8)$ and the highest mean correlation with all remaining features were removed, followed by application of the Boruta algorithm to select important features [16]. The Boruta algorithm combines feature rank based on the random forest classification algorithm and selection frequency based on multiple iterations of the feature selection procedure. Recursive feature elimination based on bagged tree models with a cross-validation technique 
TABLE 2 Laboratory results of patients with coronavirus disease 2019 at hospital admission

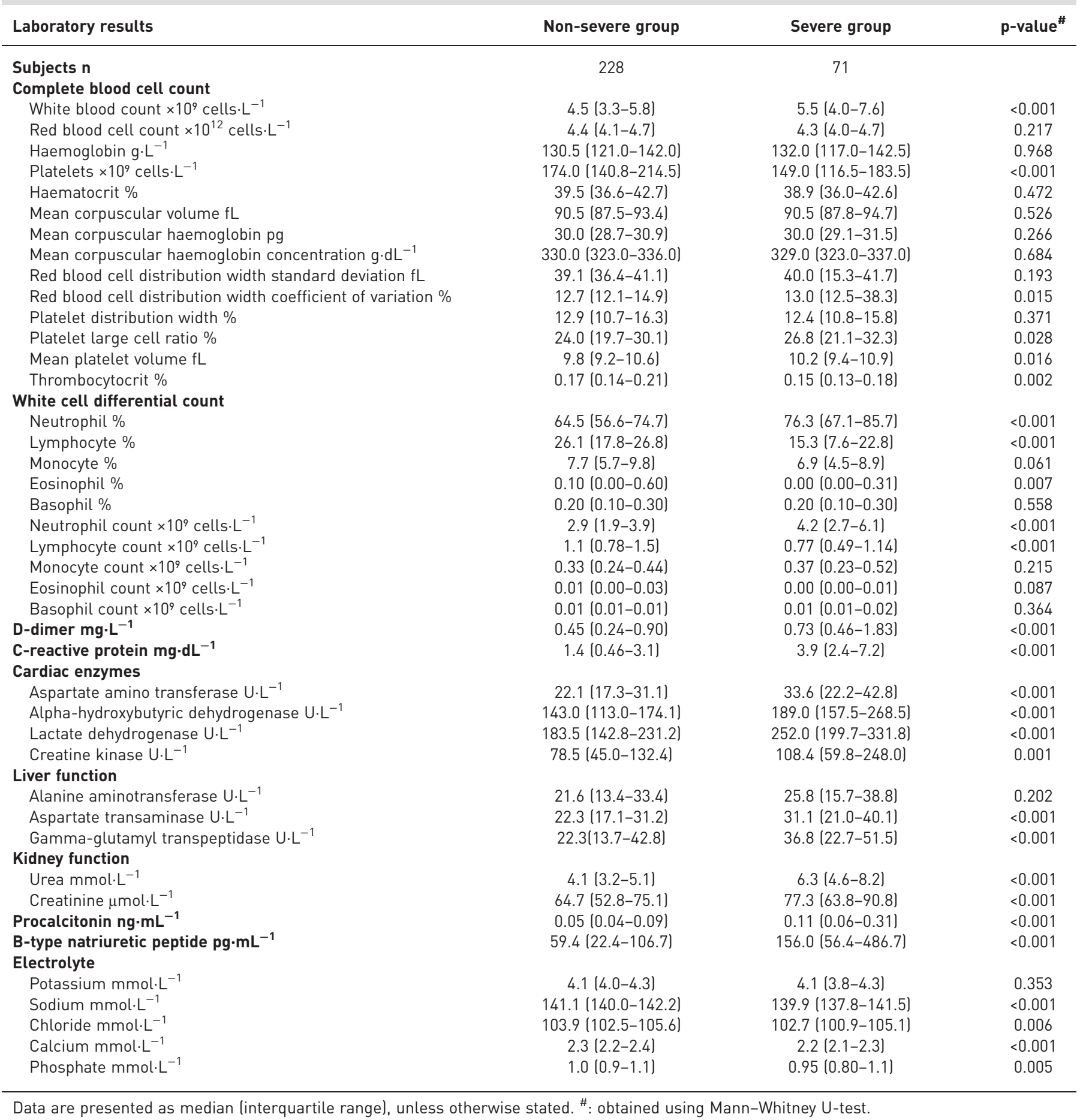

(10 folds, 10 times) was performed to select the best performing combination of features. In order to balance the positive and negative sample size, an adaptive synthetic sampling approach for imbalanced learning (ADASYN) was used during feature selection and modelling [17]. The feature selection process was used for clinical, laboratory and CT semantic models alone, and in combination.

Logistic regression models based on selected features were trained and the validation dataset was used to internally validate the prognostic performance of the models. Four models were trained: model 1 contained only baseline clinical features without symptoms; model 2 used all selected clinical features; 

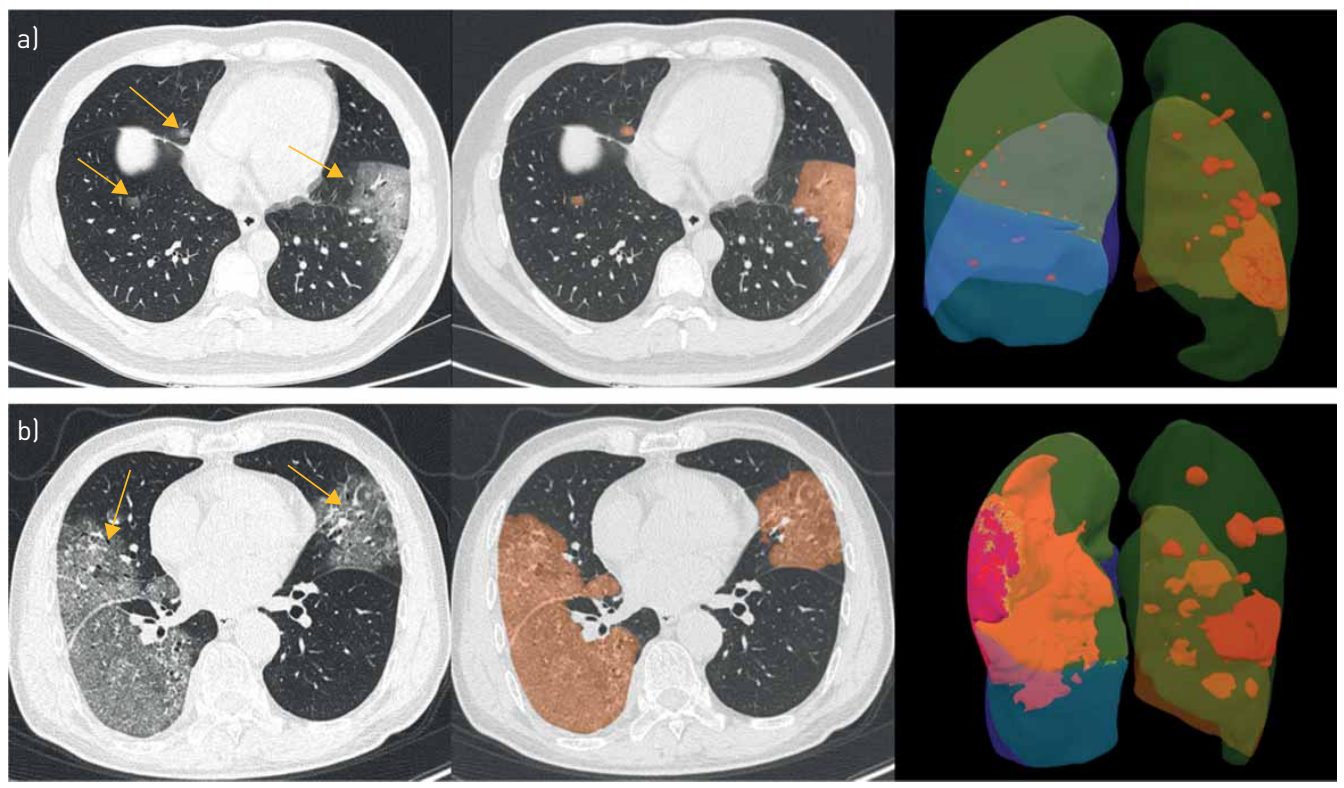

FIGURE 2 Chest computed tomography images of two patients with coronavirus disease 2019 pneumonia. a) A 48-year-old man, the focal ground-glass opacities in the bilateral lung lobes (yellow arrow) were automatically segmented (orange areas) and the lesion volume in each lobe was calculated: right superior lobe: $0.2 \%$; right middle lobe: $0.3 \%$; right inferior lobe: $0.1 \%$; left superior lobe: $0.9 \%$; and left inferior lobe: $9.4 \%$. The lesion range score was $6(1+1+1+1+2)$. b) A 70 -year-old man, the peripheral ground-glass opacities in the bilateral lung lobes (yellow arrow) were automatically segmented (orange areas) and the lesion volume in each lobe was calculated: right superior lobe: $32.1 \%$; right middle lobe: $16.4 \%$; right inferior lobe: $62.7 \%$; left superior lobe: $12.8 \%$; and left inferior lobe: $7.3 \%$. The lesion range score was $13(3+2+4+2+2)$.

model 3 used selected semantic CT features, age and sex; and model 4 employed all selected clinical, laboratory and CT features.

The prognostic performances of the best model were compared with other models on the training dataset, due to a bigger sample size. The performance of the best model and PSI scoring were gauged on the datasets via the receiver operator characteristic (ROC) and confusion matrix. In order to gauge the level of overfitting, the outcomes were randomised on the best model and the entire process repeated, from feature selection to model building and evaluation.

The patients from the training datasets were divided into low, medium and high risk according to the first quartile (25th percentile) and the third quartile (75th percentile) of probabilities from the best performing model. Nomograms and online calculators were used to provide the interpretability of the best trained models. The test datasets were used to gauge the prognostic performance and the validity for the best model.

\section{Statistical analysis}

Baseline data were summarised as median, and categorical variables as frequency (\%). Differences between the severe group and the non-severe group were tested using the Mann-Whitney test for continuous data and Fisher's exact test for categorical data. Feature correlations were measured using the Spearman correlation coefficient. We determined the area under the ROC curve (AUC) with its $95 \% \mathrm{CI}$ and tested AUC difference between models 1-3 and model 4 using the method of DELong et al. [18], measures of prognostic performance including the AUC and metrics derived from the confusion matrix accuracy, sensitivity, specificity, positive prediction value (PPV) and negative prediction value (NPV). A calibration-plot based on the Hosmer-Lemeshow test was used to estimate the goodness-of-fit and consistency of the model on the test datasets. All p-values were two-sided and $\mathrm{p}<0.05$ was regarded as significant. All statistical analyses, modelling and plotting were performed in R (version 3.5.3), and the detailed package characteristics are listed in table S1.

\section{Results}

\section{Demographic and clinical characteristics}

Out of 299 hospitalised COVID-19 patients in the retrospective cohort, the median (interquartile range) age was $50(35.5-63.0)$ years and age range was 20-94 years. $137(45.8 \%)$ were male. All the clinical characteristics and CT findings are summarised in table 1 , and more details of laboratory findings can be 
seen in table 2. Out of 426 hospitalised COVID-19 patients in five cohorts as test datasets, the median (interquartile range) age was 62 (50.0-72.0) years and age range was 19-94 years. 236 (55.4\%) were male.

\section{Feature selection}

Among the clinical features, age, hospital employment, body temperature and the time of onset to admission were selected. Lymphocyte (proportion), neutrophil, (proportion), CRP, lactate dehydrogenase $(\mathrm{LDH})$, creatine kinase, urea and calcium were selected from the laboratory feature set. Only the lesion range score was selected from CT semantic features. When putting these three category features together to select features, age, lymphocyte (proportion), CRP, LDH, creatine kinase, urea and calcium were finally included in the combination model.

\section{Models performance in the training and validation dataset}

Model performance was as follows. Model 1 was based on age and hospital employment and showed an AUC of 0.74 (95\% CI $0.69-0.79)$ on the training dataset and 0.83 (95\% CI 0.72-0.94) on the validation dataset. Model 2 with the clinical features of age, hospital employment, body temperature and the time of onset yielded an AUC of 0.78 (95\% CI 0.73-0.83) on the training dataset and 0.74 (95\% CI, 0.59-0.89) on the validation dataset. Model 3 was based on age and lesion range score on CT and had an AUC of 0.75 (95\% CI 0.70-0.80) on the training dataset and 0.83 (95\% CI, 0.72-0.94) on the validation dataset.

When pooling these three categories of features, the combination model (model 4) selected seven features (age, lymphocyte (proportion), CRP, LDH, creatine kinase, urea and calcium), which achieved the highest AUC of 0.86 (95\% CI $0.82-0.90)$ on the training dataset and a 0.90 (95\% CI $0.82-0.98)$ on the validation dataset. The AUC value of model 4 was significantly higher than model $1(p=0.001)$, model $2(p=0.033)$ and model 3 ( $\mathrm{p}=0.003)$ on the training dataset. The cut-off values from reclassification of low-, mediumand high-risk probabilities were 0.21 and 0.80 .

\section{External validation}

Model 4 was validated on the five test datasets, which showed AUCs ranging from 0.84 to 0.93 with accuracies ranging from $74.4 \%$ to $87.5 \%$, sensitivities ranging from $75.0 \%$ to $96.9 \%$, specificities ranging from $55.0 \%$ to $88.0 \%$, PPVs ranging from $66.7 \%$ to $84.1 \%$, and NPVs ranging from $73.9 \%$ to $95.7 \%$ (table 3). The ROC, confusion matrix and calibration plots are shown in figure 3. The results of randomising the outcomes and re-running the analysis yielded an AUC of 0.50 (95\% CI 0.44-0.55) for model 4.

\section{Clinical use}

Based on the selected features from the best models, a nomogram was established to quantitatively assess the severity risk of illness (figure 4). The developed online calculators can be found at www.covid19risk.ai. Compared to PSI scoring (cut-off value: 90), model 4 (cut-off value: 0.53) showed higher AUCs, accuracies, sensitivities and NPVs on the five test datasets (table 3). There was significant difference for the proportion of severe patients among low-, medium- and high-risk groups in the five test datasets (figure 5).

\section{TABLE 3 The prognostic performance of the combination model (model 4) on five test datasets}

\begin{tabular}{|c|c|c|c|c|c|c|c|}
\hline Dataset & Tool & AUC $(95 \% \mathrm{CI})$ & Accuracy $(95 \%$ CI) \% & Sensitivity \% & Specificity \% & PPV \% & NPV \% \\
\hline Test 1 & PSI & $0.87(0.74-1.0)$ & $77.8(60.9-89.9)$ & 56.3 & 95.0 & 90.0 & 73.1 \\
\hline Test 2 & PSI & $0.86(0.79-0.94)$ & $65.6(54.8-75.3)$ & 38.8 & 97.6 & 95.0 & 57.1 \\
\hline \multirow[t]{2}{*}{ Test 3} & Model & $0.93(0.83-1.0)$ & $87.5(71.0-96.5)$ & 85.7 & 88.0 & 66.7 & 95.7 \\
\hline & PSI & $0.89(0.77-1.0)$ & $75.0(56.6-88.5)$ & 0.00 & 96.0 & 0.00 & 77.4 \\
\hline \multirow[t]{2}{*}{ Test 5} & Model & $0.89(0.85-0.94)$ & $79.2(72.5-84.9)$ & 96.9 & 57.5 & 73.6 & 93.9 \\
\hline & PSI & $0.71(0.63-0.78)$ & $62.9(55.4-70.0)$ & 42.9 & 87.5 & 80.8 & 55.6 \\
\hline \multirow[t]{2}{*}{ Mean (Test 1-5) } & Model & 0.88 & 80.1 & 84.6 & 73.7 & 75.2 & 85.2 \\
\hline & PSI & 0.82 & 69.8 & 40.0 & 89.9 & 67.2 & 65.8 \\
\hline
\end{tabular}

PSI: pneumonia severity index; AUC: area under the receiver operating characteristic curve; PPV: positive prediction value; NPV: negative prediction value. 

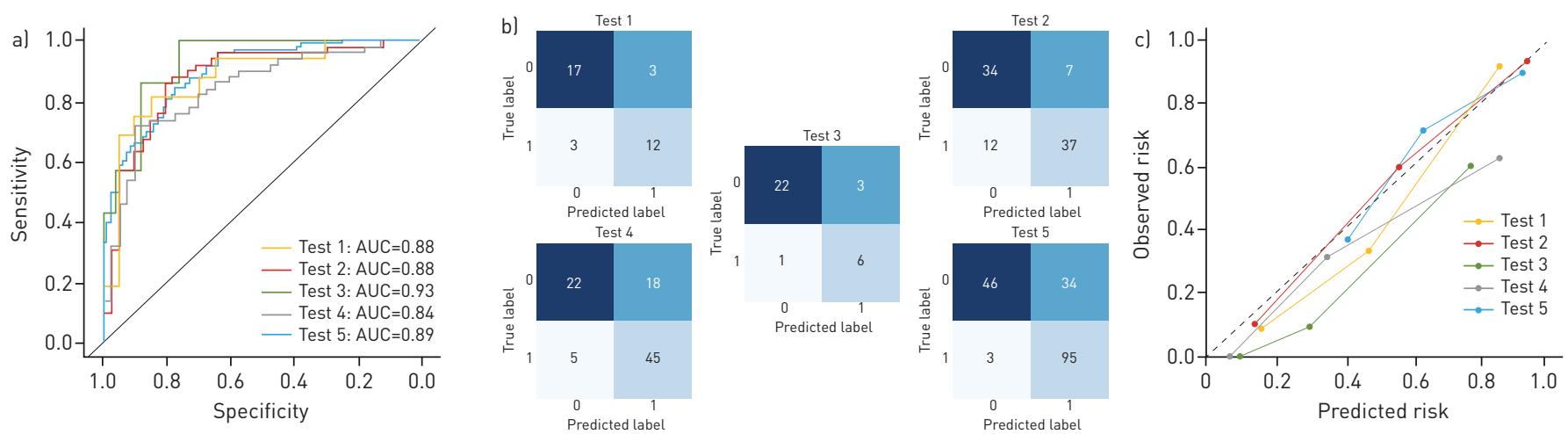

FIGURE 3 The a) receiver operator characteristic curve, b) confusion matrix and cl calibration curve for the test datasets. AUC: area under the curve.

\section{Discussion}

This international multicentre study analysed individually and in combination, clinical, laboratory and radiological characteristics for COVID-19 patients at hospital admission to retrospectively develop and prospectively validate a prognostic model and tool to assess the severity of the illness, and its progression, and to compare these with PSI scoring. We found that COVID-19 patients who developed a severe illness were often of an advanced age, had multiple comorbidities, presented with chest tightness, and had abnormal laboratory results and broader lesion range on lung CT on admission. Using simpler linear regression models yielded better prognostic performance than PSI scoring in the test datasets. We believe these models could be useful for risk assessment and triage.

Previous studies have reported that age and underlying comorbidities (such as hypertension, diabetes and cardiovascular diseases) may be risk factors for the COVID-19 patients requiring ICU admission [19, 20].

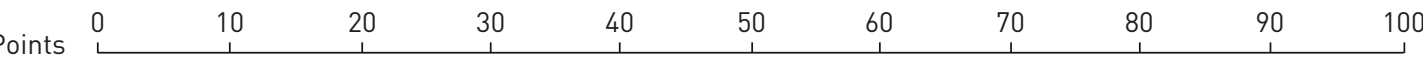

$\begin{array}{lllllllllll}\text { Age years } & 1 & 1 & 1 & 1 & 1 & 1 & 1 & 1 & 1 & 10 \\ & 20 & 30 & 40 & 50 & 60 & 70 & 80 & 90 & 100\end{array}$

$\begin{array}{lllllllllll}\text { Lymphocytes } \% & 90 & 80 & 70 & 60 & 50 & 40 & 30 & 20 & 10 & 0\end{array}$

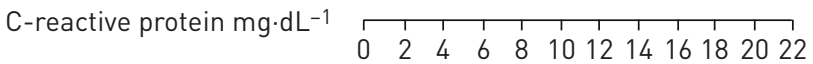

Lactate deydrogenase U.L-1

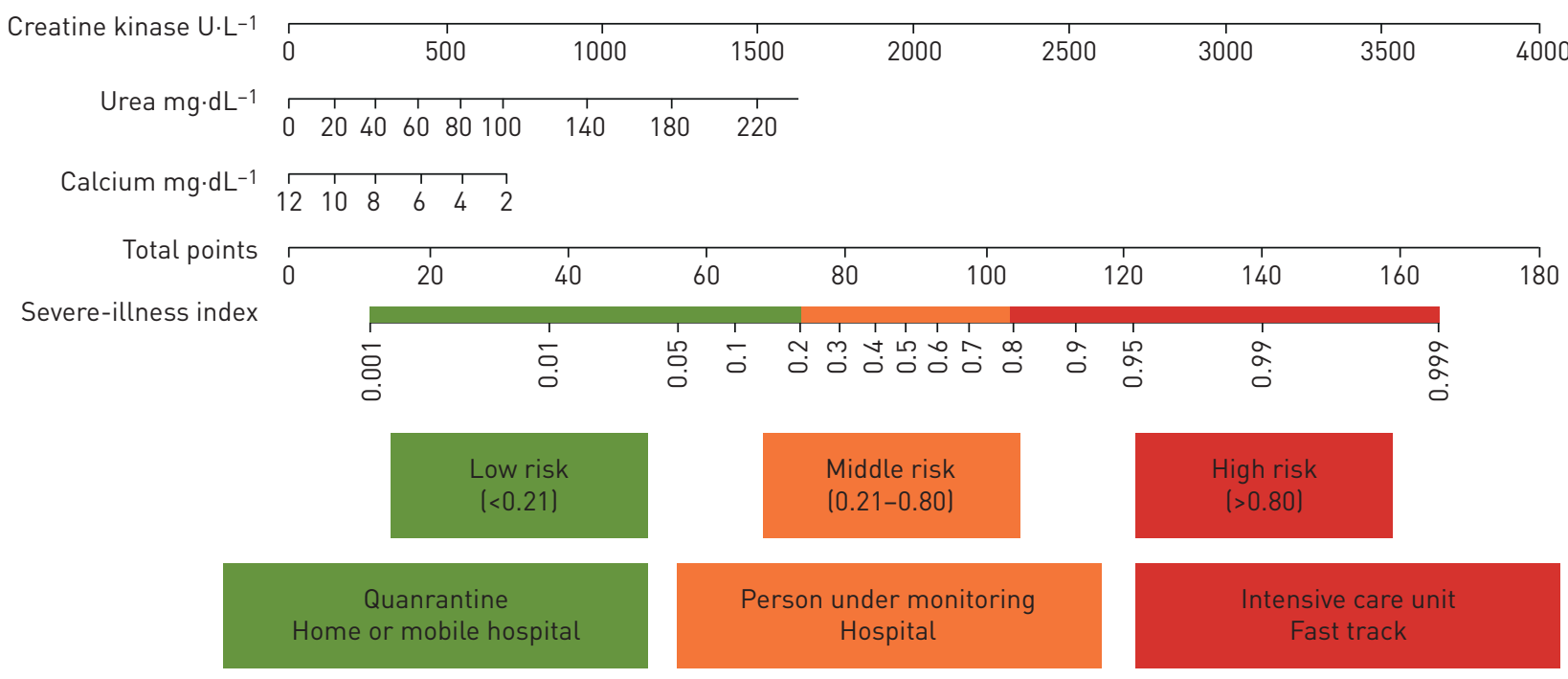

FIGURE 4 Severe-illness risk nomogram and triage tool for clinicians. 
FIGURE 5 Histogram plot of the proportion of severe patients in low-, medium- and high-risk groups of the test datasets. $* * *$ : $p<0.001 ;{ }^{\#}: p=0.006$.
Low-risk group $\square$ Medium-risk group $\square$ High-risk group

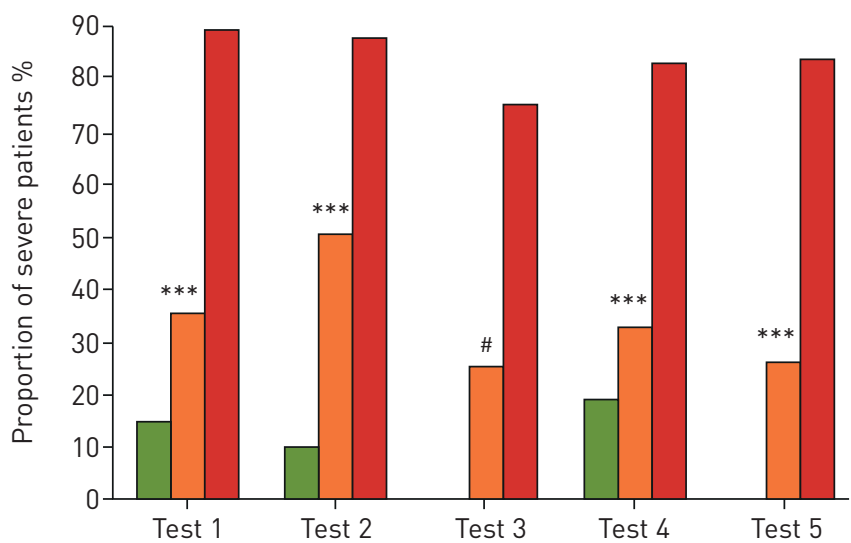

In this study, we found that the elderly COVID-19 patients who were male, non-hospital staff, suffering from hypertension, diabetes, cardiopathy disease, COPD, cerebrovascular disease, renal disease, hepatitis B virus infection, lower body temperature and chest tightness were more vulnerable to develop a severe illness in the early stages of the disease. Among these features, age, hospital staff, body temperature and the time of onset to admission had certain prognostic abilities. Age was the most important feature, which may interact with other features, which was why only age was selected into our combination model (model 4) from these features. ZHou et al. [21] have confirmed that SARS-CoV-2 uses the same cell entry receptor (angiotensin-converting enzyme-2) as SARS-CoV. However, whether COVID-19 patients with hypertension and diabetes have higher severe illness risk, which is due to treatment with angiotensin-converting enzyme-2, increasing drugs is still unknown [22]. Hospital staff had a lower risk of progression, possibly due lower age, higher levels of education, and more medical knowledge once infected, although the unbalanced nature of this type of data has to be taken into account.

Furthermore, early studies have shown that COVID-19 patients with severe illness had more laboratory abnormalities such as CRP, D-dimer, lymphocytes, neutrophils and LDH, than those patients with non-severe illness, which were associated with the prognosis $[19,20,23]$. In our study, we also found that the severe group had numerous laboratory abnormalities in complete blood cell count, white cell differential count, D-dimer, CRP, liver function, renal function, procalcitonin, B-type natriuretic peptides and electrolytes. Among these abnormalities, lymphocyte proportion, neutrophil proportion, CRP, LDH, creatine kinase, urea and calcium were significant prognostic factors, which suggest that COVID-19 may cause damage to multiple organ systems when developing into a severe illness. However, current pathological findings of COVID-19 suggest that there is no evidence that SARS-CoV-2 can directly impair the other organs such as liver, kidney and heart [24].

Current reports have shown that thin-slice chest CT is a powerful tool in clinical diagnosis due to the high sensitivity and the ability to monitor the development of the disease $[25,26]$. In addition, a previous study reported that ground-glass opacity and consolidation were the most common CT findings for COVID-19 patients with pneumonia, while being nonspecific [27]. Clinical observations showed that there were significantly more consolidation lesions in ICU patients on admission, while more ground-glass opacity lesions were observed in non-ICU patients [28]. In our study, we found that vascular enlargement, air bronchogram and lesion range score differed significantly between non-severe and severe groups. Among these features, only the lesion range score had prognostic power, but not enough to be selected for the combination model. This indicates that while these early stage CT semantic features could have diagnostic value, they have limited ability to prognose the onset of severe illness in COVID-19 patients.

The Chinese National Health Committee added some warning indicators for severe or critical cases in the updated diagnosis and treatment plan for COVID-19 patients (version 7) [29], which includes progressive reduction of peripheral blood lymphocytes, a progressive increase of interleukin-6, CRP and lactate, and rapid progression of lung CT findings in a short period. In this study, we used age, lymphocyte fraction, CRP, LDH, creatine kinase, urea and calcium scores from clinical, laboratory, and radiological examinations recorded at hospital admission to train a model for the prediction of the onset of severe illness. Our model combining these features from multiple sources showed a favourable performance when validated in the five external datasets from China, Italy and Belgium. In addition, the model is able to stratify COVID-19 patients into low-, medium- and high-risk groups for developing severe illness. We propose that this model, with its higher prediction performance and simplicity than PSI score, could be 
used for a preliminary screening and triage tool at hospital admission for the potential to develop severe illness. Furthermore, the model could be used for the selection and/or stratification of patients in clinical trials in order to homogenise the patient population. Follow-up laboratory tests are needed to assess the severity risk with a higher accuracy.

As one of the coronavirus family's infecting humans, SARS-CoV-2 has similar aetiologic, clinical, radiological and pathological features to those of severe acute respiratory syndrome coronavirus and Middle East Respiratory Syndrome coronavirus [23, 30, 31]. Therefore, we believe that developing a reliable early warning model based on present clinical, radiological and pathological data is necessary for current outbreaks and possible future outbreaks of coronaviruses.

Our study has several limitations. First, selection bias is unavoidable as is the limited and unbalanced sample size. Secondly, patients from different races and ethnicities may have diverse clinical and laboratory results, and the self-medication of patients before admission may affect the clinical and laboratory results. Thirdly, the threshold to go to the hospital and hospitalisation management can vary from country to country, and we are also aware that RNA viruses can mutate rapidly and could have an impact of the performance of the models. We therefore propose that those models should be continuously updated to achieve a better performance for example using privacy preserving distributed learning approaches [32, 33]. Fourthly, the CT features used for this study are semantic features from the first CT scan, and radiomics or deep learning approaches may improve its prognostic performance, and follow-up CT scans may yield more information. Finally, due to the large number of predictors included in the analysis, and the complexity of feature selection and modelling, overfitting is always possible. We have mitigated this with the use of external validation cohorts, and by re-running the analysis on randomised outcomes to arrive at a "chance" (AUC=0.5) result.

\section{Conclusions}

Elderly COVID-19 patients and non-hospital staff seem more vulnerable to develop a severe illness after hospitalisation as per defining criteria, which can cause a wide range of laboratory and CT anomalies. Furthermore, our model based on lactate dehydrogenase, C-reactive protein, calcium, age, lymphocyte proportion, urea and creatine kinase might be a more useful preliminary screening and triage tool than pneumonia severity index for risk assessment of COVID-19 patients at hospital admission.

Author contributions: G. Wu, P. Yang, Y. Xie, X. Wang, and P. Lambin conceived and designed the study. G. Wu and P. Yang contributed to the literature search. P. Yang, X. Rao, J. Li, J. Li, D. Du, S. Zhao, Y. Ding, B. Liu, W. Sun, F. Albarello, A. D’Abramo, V. Schininà, E. Nicastri, J. Wu, M. Occhipinti, G. Barisione, E. Barisione, J. Guiot, A. Frix, M. Moutschen, R. Louis, P. Lovinfosse, and C. Yan contributed to data collection. G. Wu, H. Woodruff, and P. Lambin contributed to data analysis. G. Wu, H. Woodruff, and P. Lambin contributed to data interpretation. G. Wu and C. Yan contributed to the tables and figures. G. Wu, I. Halilaj, and P. Lambin contributed to build a website. G. Wu, P. Yang, $\mathrm{H}$. Woodruff, and P. Lambin contributed to writing of the report.

Conflict of interest: G. Wu has nothing to disclose. P. Yang has nothing to disclose. Y. Xie has nothing to disclose. H.C. Woodruff owns shares in Oncoradiomics, outside the submitted work. X. Rao has nothing to disclose. J. Guiot has nothing to disclose. A-N. Frix has nothing to disclose. R. Louis has nothing to disclose. M. Moutschen has nothing to disclose. Jiawei Li has nothing to disclose. Jing Li has nothing to disclose. C. Yan has nothing to disclose. D. Du has nothing to disclose. S. Zhao has nothing to disclose. Y. Ding has nothing to disclose. B. Liu has nothing to disclose. W. Sun has nothing to disclose. F. Albarello has nothing to disclose. A. D'Abramo has nothing to disclose. V. Schininà has nothing to disclose. E. Nicastri has nothing to disclose. M. Occhipinti reports grants from Menarini Foundation and Novartis, outside the submitted work. G. Barisione has nothing to disclose. E. Barisione has nothing to disclose. I. Halilaj has nothing to disclose. P. Lovinfosse has nothing to disclose. X. Wang has nothing to disclose. J. Wu has nothing to disclose. P. Lambin reports, within the submitted work, minority shares in The Medical Cloud Company and, outside the submitted work, grants/sponsored research agreements from Varian medical, Oncoradiomics, ptTheragnostic/DNAmito and, Health Innovation Ventures; he received an advisor/presenter fee and/or reimbursement of travel costs/external grant writing fee and/or in kind manpower contribution from Oncoradiomics, BHV, Varian, Elekta, ptTheragnostic and Convert pharmaceuticals; P. Lambin has shares in the company Oncoradiomics SA, Convert pharmaceuticals SA and is co-inventor of two issued patents with royalties on radiomics (PCT/NL2014/050248, PCT/ NL2014/050728) licensed to Oncoradiomics and one issued patent on mtDNA (PCT/EP2014/059089) licensed to ptTheragnostic/DNAmito, three non-patented invention (softwares) licensed to ptTheragnostic/DNAmito, Oncoradiomics and Health Innovation Ventures and three non-issues, non licensed patents on Deep Learning-Radiomics and LSRT (N2024482, N2024889, N2024889).

Support statement: This work was supported from ERC advanced grant (ERC-ADG-2015, number 694812 Hypoximmuno), European Program H2020 (ImmunoSABR - number 733008, PREDICT - ITN - number 766276, CHAIMELEON - number 952172, EuCanImage - number 952103), TRANSCAN Joint Transnational Call 2016 (JTC2016 "CLEARLY" - number UM 2017-8295), China Scholarships Council (number 201808210318), and Interreg V-A Euregio Meuse-Rhine ("Euradiomics" - number EMR4). The funders had no role in the design and conduct of the study; collection, management, analysis, and interpretation of the data; preparation, review, or approval of the manuscript; and decision to submit the manuscript for publication. This work was supported by the Dutch Cancer Society (KWF Kankerbestrijding), Project number 12085/2018-2. Funding information for this article has been deposited with the Crossref Funder Registry. 


\section{References}

1 World Health Organization. Emergencies. Disease outbreaks. www.who.int/emergencies/diseases/ Date last accessed: 7 March 2020.

2 World Health Organization. Coronavirus disease 2019 (COVID-19). Situation Report - 116. www.who.int/ emergencies/diseases/novel-coronavirus-2019/situation-reports Date last updated: 15 May 2020; date last accessed: 15 May 2020.

3 World Health Organization. Events as they happen. Rolling updates on coronavirus disease (COVID-19). www.who.int/emergencies/diseases/novel-coronavirus-2019/events-as-they-happen Date last updated: 29 June 2020; date last accessed: 15 March 2020.

4 World Health Organization. Report of the WHO-China Joint Mission on Coronavirus Disease 2019 (COVID-19). www.who.int/docs/default-source/coronaviruse/who-china-joint-mission-on-covid-19-final-report.pdf Date last updated: 16-24 February 2020; date last accessed: 7 March 2020.

5 Young BE, Ong SWX, Kalimuddin S, et al. Epidemiologic features and clinical course of patients infected with SARS-CoV-2 in Singapore. JAMA 2020; 323: 1488-1494.

6 Guan WJ, Ni ZY, Hu Y, et al. Clinical characteristics of coronavirus disease 2019 in China. N Engl J Med 2020; 382: $1708-1720$.

7 Zou XJ, Li SS, Fang MH, et al. Acute physiology and chronic health evaluation II score as a predictor of hospital mortality in patients of coronavirus disease 2019. Critical Care Medicine 2020; 48: e657-e665.

8 Fine MJ, Auble TE, Yealy DM, et al. A prediction rule to identify low-risk patients with community-acquired pneumonia. N Engl J Med 1997; 336: 243-250.

9 Fralick M, Colak E, Mamdani M. Machine learning in medicine. N Engl J Med 2019; 380: 2588-2589.

10 Lambin P, Leijenaar RTH, Deist TM, et al. Radiomics: the bridge between medical imaging and personalized medicine. Nat Rev Clin Oncol 2017; 14: 749-762.

11 Wu JT, Leung K, Leung GM. Nowcasting and forecasting the potential domestic and international spread of the 2019-nCoV outbreak originating in Wuhan, China: a modelling study. Lancet 2020; 395: 689-697.

12 Bossuyt PM, Reitsma JB, Bruns DE, et al. Towards complete and accurate reporting of studies of diagnostic accuracy: the STARD initiative. Standards for Reporting of Diagnostic Accuracy. Clin Chem 2003; 49: 1-6.

13 Metlay JP, Waterer GW, Long AC, et al. Diagnosis and treatment of adults with community-acquired pneumonia. An official clinical practice guideline of the American Thoracic Society and Infectious Diseases Society of America. Am J Respir Crit Care Med 2019; 200: e45-e67.

14 International Severe Acute Respiratory and emerging Infection Consortium. Covid-19 Clinical Research Resources. https://isaric.tghn.org/covid-19-clinical-research-resources Date last accessed: 7 March 2020.

15 LabCorp. SI Unit Conversion Table. www.labcorp.com/resource/si-unit-conversion-table Date last accessed: 7 March 2020.

16 Kursa MB, Rudnicki WR. Feature selection with the boruta package. J Stat Softw 2010; 36: 1-13.

$17 \mathrm{He}$ H, Bai Y, Garcia EA, et al. ADASYN: Adaptive synthetic sampling approach for imbalanced learning. 2008, IEEE International Joint Conference on Neural Networks; pp. 1322-1328.

18 DeLong ER, DeLong DM, Clarke-Pearson DL. Comparing the areas under two or more correlated receiver operating characteristic curves: a nonparametric approach. Biometrics 1988; 44: 837-845.

19 Huang C, Wang Y, Li X, et al. Clinical features of patients infected with 2019 novel coronavirus in Wuhan, China. Lancet 2020; 395: 497-506.

20 Wang $\mathrm{D}, \mathrm{Hu}$ B, $\mathrm{Hu}$ C, et al. Clinical characteristics of 138 hospitalized patients with 2019 novel coronavirus-infected pneumonia in Wuhan, China. JAMA 2020; 323: 1061-1069.

21 Zhou P, Yang XL, Wang XG, et al. A pneumonia outbreak associated with a new coronavirus of probable bat origin. Nature 2020; 579: 270-273.

22 Fang L, Karakiulakis G, Roth M. Are patients with hypertension and diabetes mellitus at increased risk for COVID-19 infection? Lancet Respir Med 2020; 8: e21.

23 Cao B, Wang Y, Wen D, et al. A trial of lopinavir-ritonavir in adults hospitalized with severe Covid-19. $N$ Engl $J$ Med 2020; 382: 1787-1799.

$24 \mathrm{Xu} \mathrm{Z}$, Shi L, Wang Y, et al. Pathological findings of COVID-19 associated with acute respiratory distress syndrome. Lancet Respir Med 2020; 8: 420-422.

25 Song F, Shi N, Shan F, et al. Emerging 2019 novel coronavirus (2019-nCoV) pneumonia. Radiology 2020; 295: 210-217.

26 Wang Y, Dong C, Hu Y, et al. Temporal changes of CT findings in 90 patients with COVID-19 pneumonia: a longitudinal study. Radiology 2020; 296: e55-e64.

27 Chung M, Bernheim A, Mei X, et al. CT imaging features of 2019 novel coronavirus (2019-nCoV). Radiology 2020; 295: 202-207.

28 Shi H, Han X, Jiang N, et al. Radiological findings from 81 patients with COVID-19 pneumonia in Wuhan, China: a descriptive study. Lancet Infect Dis 2020; 20: 425-434.

29 National Health Commission of the People's Republic of China. Diagnosis and Treatment Protocol for COVID 19 (Trial Version 7). http://en.nhc.gov.cn/ Date last accessed: 7 March 2020.

30 Chan JWM, Ng CK, Chan YH, et al. Short term outcome and risk factors for adverse clinical outcomes in adults with severe acute respiratory syndrome (SARS). Thorax 2003; 58: 686-689.

31 Memish ZA, Perlman S, Van Kerkhove MD, et al. Middle East respiratory syndrome. Lancet 2020; 395: 1063-1077.

32 Zerka F, Barakat S, Walsh S, et al. Systematic review of privacy-preserving distributed machine learning from federated databases in health care. JCO Clin Cancer Inform 2020; 4: 184-200.

33 Deist TM, Dankers FJWM, Ojha P, et al. Distributed learning on $20000+$ lung cancer patients - the Personal Health Train. Radiother Oncol 2020; 144: 189-200. 